This document is confidential and is proprietary to the American Chemical Society and its authors. Do not copy or disclose without written permission. If you have received this item in error, notify the sender and delete all copies.

\title{
Conformational Changes and Flexibility of DNA Devices Observed by Small-Angle X-Ray Scattering
}

\begin{tabular}{|r|l|}
\hline Journal: & Nano Letters \\
\hline Manuscript ID & nl-2016-01338d.R1 \\
\hline Manuscript Type: & Communication \\
\hline Complete List of Authors: & $\begin{array}{l}\text { Bruetzel, Linda; LMU Munich, Department of Physics } \\
\text { Gerling, Thomas; TU Munich, Department of Physics } \\
\text { Sedlak, Steffen; LMU Munich, Department of Physics } \\
\text { Walker, Philipp; LMU Munich, Department of Physics } \\
\text { Zheng, Wenjun; University at Buffalo, Physics } \\
\text { Dietz, Hendrik; Technische Universität München, Physics Department } \\
\text { Lipfert, Jan; Ludwig-Maximilian-University, Department of Physics }\end{array}$ \\
\hline
\end{tabular}

\section{SCHOLARONE ${ }^{\text {m }}$ \\ Manuscripts}




\section{Conformational Changes and Flexibility of DNA Devices Observed by Small-Angle X-Ray Scattering}

Linda K. Bruetzel ${ }^{\dagger}$, Thomas Gerling ${ }^{\ddagger}$, Steffen M. Sedlak ${ }^{\dagger}$, Philipp Walker ${ }^{\dagger}$, Wenjun Zheng ${ }^{\S}$, Hendrik Dietz ${ }^{\ddagger}$, Jan Lipfert ${ }^{\dagger}$ **

†Department of Physics, Nanosystems Initiative Munich, and Center for Nanoscience, LMU Munich, Amalienstrasse 54, 80799 Munich, Germany

†Physik Department, Walter Schottky Institute, Technische Universität München, Am Coulombwall 4a, 85748 Garching near Munich, Germany

${ }^{\S}$ Physics Department, State University of New York at Buffalo, Buffalo, NY, USA

${ }^{*}$ Corresponding author: Jan Lipfert; Email: Jan.Lipfert@1mu.de; Phone: +49-89-2180-2005 


\begin{abstract}
Self-assembled DNA origami nanostructures enable the creation of precisely defined shapes at the molecular scale. Dynamic DNA devices that are capable of switching between defined conformations could afford completely novel functionalities for diagnostic, therapeutic, or engineering applications. Developing such objects benefits strongly from experimental feedback about conformational changes and 3D structures, ideally in solution, free of potential biases from surface attachment or labeling. Here we demonstrate that small-angle X-ray scattering (SAXS) can quantitatively resolve the conformational changes of a DNA origami two-state switch device as a function of the ionic strength of the solution. In addition, we show how SAXS data allow for refinement of the predicted idealized 3D structure of the DNA object using a normal mode approach based on an elastic network model. The results reveal deviations from the idealized design geometries that are otherwise difficult to resolve. Our results establish SAXS as a powerful tool to investigate conformational changes and solution structures of DNA origami and we anticipate our methodology to be broadly applicable to increasingly complex DNA and RNA devices.
\end{abstract}

\title{
KEYWORDS
}

DNA origami, small angle X-ray scattering, SAXS, normal modes, conformational changes

A fundamental aim of nanotechnology is to design synthetic objects that can adopt specific conformational states and carry out functions at the molecular scale, e.g. in transport, signal transduction, or molecular circuitry. Molecular self-assembly of DNA is a particularly successful approach towards creating versatile structures at the nanometer scale. ${ }^{1-4}$ When using the DNA origami technique, a several kilobase long circular single-stranded scaffold strand is folded into custom target shapes with the assistance of hundreds of short singlestranded staple strands. By exploiting the specificity of DNA base pairing, precisely controlled shapes reaching over $100 \mathrm{~nm}$ in size and molecular weights of several MDa can be created. $^{2,5-8}$

While an important initial focus in the design of self-assembled DNA structures was to create static objects of well-defined shapes, ${ }^{1-3,6}$ more complex functions require dynamic $3 \mathrm{D}$ nanostructures that can undergo controlled conformational changes. Examples of dynamic 
DNA origami structures include a DNA box with a closable $\operatorname{lid},{ }^{9}$ a DNA nanorobot, ${ }^{10}$ a reconfigurable plasmonic nanostructure, ${ }^{11}$ or a DNA tweezers. ${ }^{12}$ Such dynamic DNA structures are promising candidates for applications ranging from nano-engineering ${ }^{13,14}$ to medical diagnostics and therapeutics. ${ }^{15,16}$ An important challenge in this context is the precise control over the $3 \mathrm{D}$ shape and mechanical flexibility of the target design in solution to achieve desired functionality.

So far, structural characterization of DNA origami structures has predominantly relied on atomic force microscopy (AFM) imaging ${ }^{14,17,18}$ and negative-stain transmission electron microscopy (TEM). ${ }^{6,19-21}$ While these techniques are well suited to image static structures, they both rely on immobilizing samples on a surface and involve steps such as drying or staining the samples, which renders the solution conditions far from physiological. Cryoelectron microscopy provides less harsh conditions and has recently been successfully applied to DNA origami structures ${ }^{9,22}$ but still requires immobilized samples embedded in vitrified ice, potentially biasing the conformation of the sample and making it difficult to detect conformational changes upon variation in solution conditions.

In contrast, small-angle X-ray scattering (SAXS) can probe molecular conformations and transitions and provides low-resolution structural information on molecules and molecular assemblies in solution. ${ }^{23,24}$ As SAXS can operate under virtually arbitrary solution conditions, ${ }^{25,26}$ the technique is ideally suited to detect conformational changes triggered by changes in solution environment, such as ionic strength, denaturant, temperature, or ligand binding. SAXS has proven very powerful to detect the large structural changes associated with the folding of proteins ${ }^{27-29}$ and nucleic acids, ${ }^{30-32}$ but can also readily detect more subtle conformational changes, e.g. triggered by the binding of small-molecule ligands. ${ }^{33-36}$

Recently, Gerling et al. ${ }^{37}$ established a framework based on shape-complementary recognition for the programmable and reversible assembly and disassembly of complex 3D shapes built from DNA. One important example involves a dynamic "switch" device, where multiple weak base stacking interactions were exploited to change conformations between a closed and an open state as a function of temperature or ionic strength of the solution. Here we use small-angle X-ray scattering (SAXS) to probe the structure and conformational changes of the switch device in solution. In particular, we detect and evaluate the conformational changes upon variations in solution conditions and observe quantitative agreement with solution Förster resonance energy transfer (FRET) measurements. In addition, we refine the $3 \mathrm{D}$ structure of the switch objects against the scattering data using a normal mode based flexible fitting procedure and find evidence for swelling and structural 
rearrangements away from idealized DNA helix geometries. Together, our results establish SAXS as a powerful technique to probe the structures and conformations of DNA devices.

DNA origami switch samples based on base stacking interactions. We performed SAXS measurements on three different variants of a DNA origami switch object that is based on shape-complementarity and base stacking interactions. ${ }^{37} \mathrm{~A}$ dynamic variant of the switch (switch D) can undergo conformational changes between an $\mathrm{x}$-shaped open and a rectangularshaped closed state (Figure 1; see Supporting Information and Supplementary Figures S1-S3 for details). This variant consists of two rigid bundles of DNA double helices arranged in a honeycomb lattice that form the two arms of the structure. The arms are connected in the middle of the structure by a single Holliday junction that acts as a pivot point for the rotational degree of freedom (Figure 1). The structure of the closed state is prescribed by shape-complementary patterns of double helical protrusions (red domains, Figure 1) and recessions (blue domains, Figure 1) that can precisely dock into each other when the two arms of the switch object come close together. The closed state is stabilized by up to 16 short-range stacking interactions of the terminal bases of shape-complementary surface topographies. The conformational equilibrium sensitively depends on ambient conditions such as the salt concentration or the temperature of the solution. ${ }^{37}$ TEM images of the switch D variant show that at low salt concentrations the great majority of structures assume the open state, while at high salt concentrations switch D particles predominantly populate the closed state. ${ }^{37}$ As reference structures we employed two static variants of the switch object that are permanently locked in the open and closed states, respectively. In the static closed variant (switch $\mathrm{C}$ ) stacking interactions are replaced by stronger hybridization interactions of 3-bases-long single-stranded overhangs of corresponding staple strands holding the two arms of the switch object in the closed conformational state (Figure 1, right). In the static open variant (switch $\mathrm{O}$ ) all stacking interactions are deactivated and the two arms are connected by additional crossovers holding them at an opening angle of $\sim 90^{\circ}$ (Figure 1, left).

DNA origami structures give rise to high signal-to-noise SAXS profiles at $25-100 \mathrm{nM}$ concentrations. To estimate the minimum concentrations required for synchrotron-based SAXS measurements on our large ( 16000 nucleotides (nt) or $\sim 5 \mathrm{MDa})$ DNA origami structures, we used prior SAXS data of smaller nucleic acids in combination with extrapolation based on a scaling relationship (see Supporting Information). We analyzed the concentrations used for these SAXS measurements that resulted in a sufficient signal-to-noise ratio for structural analyses (which we loosely define as analyses that go beyond Guinier 
fitting of the lowest $q$ values) for a range of nucleic acid samples (Figure 2a, blue symbols). The dataset ranges from an $8 \mathrm{nt} \mathrm{DNA}^{38}$ to a large $(\sim 400 \mathrm{nt})$ ribozyme ${ }^{31}$ and includes both RNA $^{35,36,39,40}$ and DNA samples, ${ }^{41,42}$ as well as data for a $\sim 14$ knt DNA origami structure ${ }^{9}$ recorded at an in-house X-ray source. The data are well described by a scaling relationship of the form $c \sim M W^{v}$, where $c$ is the required concentration, $M W$ the molecular weight, and the scaling exponent $v$ was fitted to be $v=1.30$ (Figure 2a, dashed line, and Supplementary Information). The scaling relation predicts that concentrations of $\sim 10-50 \mathrm{nM}$ are sufficient to obtain a good scattering signal for a $\sim 16$ knt DNA structure. Experimentally, we indeed obtained good signal-to-noise scattering profiles for concentrations as low as $25 \mathrm{nM}$ of the DNA origami structures (Figure $2 \mathrm{~b}$ and Supplementary Figure S4), in excellent agreement with the predicted scaling relationship (Figure 2a, red star). Additional measurements at 50 and

$100 \mathrm{nM}$ concentration display even higher signal-to-noise ratios (especially in the higher $q$ range) and are superimposable after scaling by concentration, indicating the absence of aggregation, radiation damage or interparticle interference (Figure $2 \mathrm{~b}$ and Supplementary Figure S4). Interparticle interference effects occur if the particles in solutions are, on average, sufficiently close to interact, e.g. via excluded volume or electrostatic effects. We note that interparticle interference effects are expected to be (even) weaker for larger macromolecular assemblies as the typical intermolecular distances increase for higher molecular weights due to the lower required concentration (Figure 2a, inset). For instance, the average intermolecular distance of a $24 \mathrm{bp}$ DNA sample (Radius of gyration $R_{g} \sim 2.4 \mathrm{~nm}$ ) measured at a concentration of $0.2 \mathrm{mM}$ is around $20 \mathrm{~nm}$, whereas for our DNA origami objects $\left(R_{g} \sim 28 \mathrm{~nm}\right.$, see below) measured at $25 \mathrm{nM}$ it is around $400 \mathrm{~nm}$. Remarkably, due to their large size, the DNA origami objects give rise to scattering profiles with a dynamic range of $\geq 4$ orders of magnitude in intensity, with features identifiable up to $q \approx 3 \mathrm{~nm}^{-1}$. We note that while the scaling argument and extrapolation shown here only provide a rough estimate of the required sample concentrations, we anticipate that it can provide a useful guideline to other SAXS experiments on nucleic acid assemblies as well.

SAXS reveals structural features of DNA origami objects. For a first structural characterization, we analyzed the scattering data from the static switch samples, which serve as reference samples for the dynamic switch variant. When comparing the scattering profiles of the switch $\mathrm{O}$ and switch $\mathrm{C}$ samples, we observe significant differences in the $q$-range below $q<0.5 \mathrm{~nm}^{-1}$ (Figure 3a), in line with global structural differences in the open and closed 
states. For higher $q$, corresponding to smaller length scales, the scattering curves largely coincide, exhibiting two distinct peaks.

We performed a Guinier analysis of the scattering profiles in the low $q$ range to determine the overall radii of gyration $\left(R_{g}\right)$ (see Supporting Information and Supplementary Figure S5). We obtained an $R_{g}$ of $(27.9 \pm 0.1) \mathrm{nm}$ for the switch C (Table 1), which is in excellent agreement with a theoretical $R_{g}$ of $28 \mathrm{~nm}$, derived from approximating the closed switch as a rectangular beam, with $R_{g}^{\text {theo }}=1 / 3\left[(W / 2)^{2}+(H / 2)^{2}+(L / 2)^{2}\right]^{1 / 2}$, where $W, H$ and $L$ are the width, height and length of the object, respectively (Figure 1). For the switch $\mathrm{O}$ sample we found an average $R_{g}$ of $(29.0 \pm 0.2) \mathrm{nm}$ (Table 1), overall similar to switch C, which is expected as the approximate distances from the center of mass are conserved upon the transition from the closed to the open state.

For elongated rod-like particles, where the axial dimension is much larger than the radial dimension (as is the case for the DNA origami structures investigated in this work) the scattering intensity can be factorized in an axial and radial scattering component. ${ }^{43}$ Analysis of the intermediate $q$-range then permits the calculation of the radius of gyration for the radial cross-section $\left(R_{c}\right)$ (see Supporting Information). We obtained an average $R_{c}$ value for the switch $\mathrm{C}$ of $(6.7 \pm 0.1) \mathrm{nm}$ corresponding to a radius of the cross-section $R \sim 9.4 \mathrm{~nm}$, which is in good agreement with the cross-sectional dimensions of the design model (Figure 1). The switch $\mathrm{O}$ can be thought of as being assembled from two rods where the cross-section is half of the size as for the switch $\mathrm{C}$ sample. Here, a smaller average $R_{c}$ value of $4.8 \mathrm{~nm}$, corresponding to a radius $R \sim 6.8 \mathrm{~nm}$, is fully consistent with the expected reduction of the cross-sectional area when the switch changes from the closed to an open conformation.

A Kratky representation $\left(q^{2} \cdot I(q)\right.$ vs. $\left.q\right)$ of the scattering data of switch $\mathrm{C}$ and switch $\mathrm{O}$ reveals a number of peaks that can be related to structural features (Figure $3 \mathrm{~b}$ ). The peak and shoulder at lowest $q$-values ("1", Figure 3b) for the switch $\mathrm{O}$ and switch $\mathrm{C}$ samples, respectively, at $q \sim 0.06 \mathrm{~nm}^{-1}$ are related to the overall dimensions of the objects $(\mathrm{d} \sim 100 \mathrm{~nm})$ and to their $R_{g}$ via $q \approx \sqrt{3} / R_{g} \approx 0.06 \mathrm{~nm}^{-1}$. The major peaks (" 2 ", Figure $3 \mathrm{~b}$ ) at $q \approx 0.14 \mathrm{~nm}^{-1}$ and at $q \approx 0.19 \mathrm{~nm}^{-1}$ for the switch $\mathrm{C}$ and the switch $\mathrm{O}$ sample, respectively, are related to the maximum of the cross-sectional intensity expected at $q_{\max }=1 / R_{c}$. The fitted $R_{c}$ values of 6.7 $\mathrm{nm}$ for the switch $\mathrm{C}$ and $4.8 \mathrm{~nm}$ for the switch $\mathrm{O}$ sample (Table I) suggest $q_{\max } \sim 0.15 \mathrm{~nm}^{-1}$ and $q_{\max } \sim 0.2 \mathrm{~nm}^{-1}$, in very good agreement with the observed peak positions in the Kratky plot. In the higher $q$-range, both scattering profiles display a small and broad peak ("“3”, Figure $3 \mathrm{~b})$ at $q \sim 1.0 \mathrm{~nm}^{-1}(d \sim 6.3 \mathrm{~nm}$, Figure 1 red arrow $a)$ and a more pronounced peak (" 4 ", Figure $3 b)$ at $q \sim 1.6 \mathrm{~nm}^{-1}(d \sim 3.9 \mathrm{~nm}$, Figure 1 red arrow $b)$, which corresponds to the 
distances between and within the honeycomb lattice, respectively (Figure 1). These values are in approximate agreement with the theoretical values and the relative number of these distances is approximately the same for both conformations, consistent with the similarity of the scattering curves in the higher $q$-regime. We note that features relating to the structure of single DNA helices (such as their diameter, the minor groove/major groove periodicity, and the spacing between base pairs) occur on even shorter length scales and thus correspond to $q$ values $\geq 3 \mathrm{~nm}^{-1}$, which have been probed in wide-angle X-ray scattering measurements, ${ }^{44}$ but are not the focus of the present work.

To more directly visualize the contribution of features on various length scales, we calculated the pair distance distribution function $P(r)$ (see Supporting Information and Supplementary Figure S6), which describes a histogram of all pairwise distances $r$ within the sample (Figure $3 c)$. For both static open and closed structures, we find a maximum pairwise distance $D_{\max }$ of $95 \mathrm{~nm}$, in good agreement with the expected maximum distance from the designed structures. The shape of the $P(r)$ function obtained for the switch $\mathrm{C}$ variant is peaked at low $r$ with a long tail out to higher $r$, characteristic of an elongated object. In contrast, the $P(r)$ of the switch $\mathrm{O}$ exhibits an overall more Gaussian shape, characteristic of a more globular object. In the switch C $P(r)$ function, we observe a well-defined peak at an intraparticle distance of $16 \mathrm{~nm}$, which corresponds to the maximum transverse distance of the closed state (Figure 1). This peak is not apparent in the $P(r)$ function of the switch $\mathrm{O}$ sample, as expected, since the opening of the switch reduces the transverse distance to $8 \mathrm{~nm}$. For the open conformation we find a smaller feature at $12 \mathrm{~nm}$ that is related to the height of the switch object and also contains contributions from the maximum transverse distance of $\sim 8 \mathrm{~nm}$ (Figure 1), which become more exposed in the open state. The dominant $P(r)$ peak for the switch $\mathrm{O}$, however, occurs around $40 \mathrm{~nm}$, the distance associated with the length of each of the two opened arms.

Conformational populations of the dynamic switch variant. Having demonstrated that SAXS clearly reveals the large-scale conformational changes between the open and closed versions of the static switch object, we next analyzed the conformational states of the dynamic version of the switch (switch D) at high $(30 \mathrm{mM})$ and low $(5 \mathrm{mM})$ magnesium chloride $\left(\mathrm{MgCl}_{2}\right)$ concentrations (Figure $4 \mathrm{a}, \mathrm{b}$ and Supplementary Figure S8). In general, the scattering profile from an ensemble is given by the sum of the scattering profiles for the individual components, weighted by their relative occupancy. In the case of a two-state system, the scattering profile can be described by a linear superposition of the two states:

$$
I(q)=f_{1} \cdot I_{1}(q)+f_{2} \cdot I_{2}(q)
$$


$I_{1}(q)$ and $I_{2}(q)$ are the scattering profiles and the coefficients $f_{1}$ and $f_{2}$ are fractional occupancies of states 1 and 2, respectively. Using the scattering profiles of the switch $\mathrm{O}$ and switch $\mathrm{C}$ objects for the open and closed states, we fitted the scattering profiles of the dynamic variant at $30 \mathrm{mM} \mathrm{MgCl}$ (switch D30) and $5 \mathrm{mM} \mathrm{MgCl}$ (switch D05) as a linear superposition of the two states (Supplementary Figure S8). Under both conditions, the twostate fits provide an overall excellent description of the experimental data, suggesting that the conformations of the dynamic switch variant can be well approximated by a two-state model featuring the open and closed states. For both samples slight deviations of the fit become apparent at higher $q$-values, which might imply that there exist structural differences within the internal honeycomb lattice between the dynamic and static versions. This might be attributed to the different concentrations of $\mathrm{MgCl}_{2}$ in the sample solutions, which have an impact on structural integrity and flexibility owing to its efficacy in screening interhelical repulsion and stabilizing DNA Holliday junctions (see also below). ${ }^{45,46}$ In addition, previous TEM studies on the switch D05 sample revealed a slightly reduced opening angle compared to the fixed opening angle of $90^{\circ}$ for the switch $\mathrm{O}$ sample, ${ }^{37}$ which might cause some additional differences in the scattering profiles.

Complementary to analyzing $I(q)$, we applied a two-state model analogous to Equation 1 to the $P(r)$ functions (Figure 4c). We find that the $P(r)$ function of the switch D30 sample can be described very accurately by the two-state model. For the $P(r)$ function of the switch D05 sample again slight deviations between the two-state model and the data are observable, but overall the two-state description is still accurate.

The fitted parameters $f_{1}$ and $f_{2}$ in Equation 1 provide a direct measure of the relative populations of the two states. Figure $4 \mathrm{~d}$ shows the relative populations of the closed conformation determined from the scattering intensity and $P(r)$ fits (the corresponding populations of the open conformation are the complement to $100 \%)$. From the $I(q)$ fits, we find a population of $(77 \pm 1) \%$ in the closed state for the switch D30 sample, in agreement with the expectation that screening of electrostatic repulsion at high salt concentration should lead to a predominant population of the closed conformation. In contrast, the occupancies derived for the switch D05 sample are $(3 \pm 2) \%$ for the closed state, in line with the prediction that electrostatic repulsion at lower ionic strength favors the open configuration. The $P(r)$ fits gave identical results, within experimental error (Figure 4d). These findings are further supported by the fact that the fitted cross-sectional radii of gyrations of the switch $\mathrm{D}$ object in 5 and $30 \mathrm{mM} \mathrm{MgCl}_{2}$ are close to values determined for the switch $\mathrm{O}$ and switch $\mathrm{C}$ conformations, respectively (Table 1). 
The results of the SAXS analyses can be compared to data obtained from ensemble FRET measurements and TEM imaging on switch $\mathrm{D}$ particles at varying $\mathrm{MgCl}_{2}$ concentrations ${ }^{37}$ (see Supporting Information and Supplementary Figure S7). Data from solution-based ensemble FRET measurements are in good agreement, within experimental errors, with the SAXS results (Figure 4d). From TEM imaging data, higher fractional occupancies for the closed state were obtained compared to the solution-based methods: (93 \pm 1$) \%$ of the objects were identified to be in the closed state at a $\mathrm{MgCl}_{2}$ concentration of $25 \mathrm{mM}$ and $(13 \pm 2) \%$ of closed particles were found at a $\mathrm{MgCl}_{2}$ concentration of $5 \mathrm{mM}$. The deviations of the TEMdetermined fractions to the solution-based values are modest, but statistically significant for the SAXS derived values (Figure 4d) and might be related to several factors: First, for the TEM analysis switch D particles were picked from TEM images for each salt condition and manually assigned to be either open or closed; errors were determined from binomial counting statistics. This process might introduce a slight bias, as overlapping objects could not be classified and as partially closed switch objects were considered as closed. Second, TEM imaging requires immobilization of samples on a surface potentially affecting their conformation. Furthermore, the staining process for TEM imaging can alter the global shape of the particles. ${ }^{47}$ In addition, single-molecule FRET experiments, which likewise require surface immobilization of the switch D particles, gave similar results as the TEM data. ${ }^{37}$ Taken together, the data suggest that surface immobilization and/or staining might create a modest bias towards the closed conformation and can give rise to a small population of partially closed conformations, possibly due to direct surface interactions or excluded volume effects.In order to test whether the transition from the open to the closed state of the dynamic switch variant upon the addition of $\mathrm{MgCl}_{2}$ ions can be described as a two-state process, we performed SAXS experiments on switch D samples for varying $\mathrm{MgCl}_{2}$ concentrations ranging from $3 \mathrm{mM}$ to $30 \mathrm{mM}$ (Figure $4 \mathrm{e}$ ). The scattering profiles at different $\mathrm{MgCl}_{2}$ concentrations exhibit an iso-scattering point around $q \sim 0.3 \mathrm{~nm}^{-1}$ tentatively suggesting that the conformational transition can be described as a two-state process. For a more quantitative analysis, we performed a two-state fit of the scattering profiles at each $\mathrm{MgCl}_{2}$ concentration according to Equation 1 and fitted the resulting populations by a thermodynamic model (using Equations S8 and S9 in Supporting Information) assuming a linear dependence of the free energy $\Delta G$ and the ion concentration $c$. From a least squares fit we obtained for $\Delta G_{0}=1.2$ $\mathrm{kcal} / \mathrm{mol}$ at the reference ion concentration of $5 \mathrm{mM}$ and the slope $m_{c}=-0.3 \mathrm{kcal} /(\mathrm{mol} \mathrm{mM})$ in good agreement with values based on ensemble FRET measurements (Figure 4f and Supplementary Figure S7). In addition, the two state-fits yield a good fit of the full scattering 
profiles over the entire range of $\mathrm{MgCl}_{2}$ concentrations (Supplementary Figure S8). These findings show that the switch D transition from the open to the closed state can be described adequately, at least at the current level of signal-to-noise, by a two-state model employing a single open and closed conformation, without the need to introduce intermediates states or conformations.

Overall, we find quantitative agreement between SAXS and solution FRET derived population estimates and approximate agreement with the TEM derived values, confirming the switching mechanism in the dynamic switch variant. Our findings highlight the importance of solution-based techniques when performing structural characterization of complex DNA structures.

Comparison of experimental SAXS data to idealized models and model refinement. In addition to detecting conformational transitions and providing global measures of size and shape (such as $R_{g}, R_{\mathcal{c}}$, and $D_{\max }$ ) SAXS can provide information about the full 3D solution structure of macromolecules and their assemblies. ${ }^{24,48}$ Even though the resolution of SAXS experiments is typically insufficient to compute a unique structure, it is possible to test and refine structural models against experimental SAXS data. ${ }^{49-52}$ First, we compared our experimental data to scattering profiles of the switch $\mathrm{O}$ and switch $\mathrm{C}$ samples predicted from idealized atomistic models generated by $\mathrm{CanDo}^{53}$ (see Supporting Information). The computed profiles from the CanDo models reproduce the overall shape of the experimental curves and reveal similar characteristic peaks (Figure 5a,b; Supplementary Figure S9). In addition, we obtain $R_{g}$ and $R_{c}$ values from the theoretical scattering curves, which are in good agreement with the experimentally determined values (Table 1, Supplementary Table S1). However, small, but systematic deviations between the experimental and theoretical profiles are apparent. There is an additional peak in the theoretical scattering patterns for the open state at $q \sim 0.26 \mathrm{~nm}^{-1}$ and the peaks that are visible in both experimental and theoretical curves are shifted, mostly to higher $q$ in the theoretical curves compared to experiment. Furthermore, the ratios of the peak intensity values at low and high $q$ differ between the experimental and theoretical curves. In addition, we determined the $\mathrm{P}(\mathrm{r})$ functions from the theoretical data and calculated a histogram of distances directly from the atomistic model coordinates (Supplementary Figure S9). In comparison to the experimental data, the peaks are more pronounced and deviations from the experimental peak positions are observable.

We note that even though the different methods to compute scattering profiles from the structures exhibit some differences (see Supporting Information and Supplementary Figure 
S9), they do give overall very similar results and show comparable deviations from the experimental data, suggesting that the details of the scattering computations are relatively unimportant and can not explain the observed differences to the experimental data. In principle, both the hydration layer of partially ordered water molecules around a macromolecule in solution ${ }^{49,54}$ and the ion atmosphere around charged nucleic acids ${ }^{55,56}$ contribute to the scattering profile. For simple DNA duplexes, the effect of the ion atmosphere has been studied in detail and while the it is observable, the ion cloud's contribution to the scattering is pattern is relatively minor, typically increasing e.g. the radius of gyration by a few angstroms. ${ }^{55,57}$ We have performed electrostatic calculations using linearized Poisson-Boltzmann theory (Supplementary Information and Supplementary Figures S10 and S11) to compare the electrostatic potential in the vicinity of a DNA origami structure with a simple DNA duplex. Our results suggest that the electrostatic potential and, consequently, the ion density around our DNA origami structures is only slightly elevated and overall similar in magnitude and spatial extent compared to a single double-stranded DNA helix (Supplementary Figures S10 and S11), consistent with previous reports in the literature. $^{58,59}$ Taken together, these observations suggest that for the very large DNA structures considered in this work contributions from the ion atmosphere to the scattering profile are small or negligible. In addition, we tested whether altering the density of the solvent or the contrast of the hydration layer in the range of physically plausible values would explain the observed differences between the CanDo derived models and our experimental data, but again found that while changing the hydration shell gives rise to small changes in the scattering profiles, these changes are insufficient to account for the observed differences (Supplementary Figure S12).

Combined, the differences between experimental and predicted scattering profiles indicate that the switch objects adopt conformations in solutions that differ from the idealized models generated by CanDo. Such deviations have been suggested previously: Pan et al. ${ }^{53}$ found an average root mean square deviation (RMSD) of $3.2 \AA$ between the CanDo derived model and the crystal structure of a DNA tensegrity motif. In general, electrostatic repulsion between adjacent helix bundles or at crossovers resulting in the bowing out of double helical domains $^{2,4}$ can lead to local displacements of nucleobase positions. Theoretical calculations and experimental evidence based on TEM data suggest an important role of flexibility for several DNA origami structures, ${ }^{60-62}$ indicating maximum root-mean-square fluctuation amplitudes of a few nanometers. ${ }^{60}$ In addition, a cryo-EM structure of a DNA origami object 
observed deviations between the idealized structure and the experimentally determined density map. ${ }^{22}$

There is currently no established method to refine DNA origami structures quantitatively against experimental data. A considerable challenge in this regard is the large size of our switch objects that renders refinement e.g. based on all-atom molecular dynamics ${ }^{61,63}$ challenging. As a computationally tractable approach, we turned to normal mode refinement of the CanDo derived model against the experimental SAXS data using an elastic network model. Normal mode analysis ${ }^{64-68}$ based on coarse-grained elastic network models has proven to describe large-scale conformational changes surprisingly well as compared to considerably more complex approaches ${ }^{66}$ and has been applied to deform macromolecular structures to fit and refine experimental data from cryo-EM, ${ }^{69}$ X-ray crystallography, ${ }^{67,70}$ and SAXS data. ${ }^{71-73}$ We iteratively refined the switch $\mathrm{C}$ and switch $\mathrm{O}$ structures against the experimental SAXS data by normal mode based deformations (see Supporting Information). The resulting structures yield significantly better fits to the data (Figure 5a,b): the goodness-of-fit statistic $\mathrm{X}^{2}$ (defined in Equation S4 in the Supporting Information) is reduced from $0.5 \%$ to $0.06 \%$ and from $6.7 \%$ to $1.9 \%$ for the switch $\mathrm{C}$ and switch $\mathrm{O}$ structures, respectively. We find that for the refined structures the highly symmetric lattice structure is significantly deformed (Figure 5c,d). In comparison to the initial models, some parts in the refined closed and opened switch objects swell and bulge out. This effect is especially pronounced in double helices around the center of the structure, where the two arms are connected to each other (Supplementary Figure S13). In addition, the refined structures show the helices at the ends and sides of the arms slightly bend outwards (Supplementary Figure S13). Interestingly, these effects are more pronounced in the switch $\mathrm{C}$ compared to switch $\mathrm{O}$ structure. The RMSD for the refined switch $\mathrm{C}$ structure compared to the initial model is $22.3 \AA$; for the switch $\mathrm{O}$, the refined structure has an RMSD of $8.4 \AA$ relative to the starting model. The larger deformations in the switch $\mathrm{C}$ object compared to switch $\mathrm{O}$ might be due to the more compact structure and, therefore, higher charge density, that would make electrostatic repulsion more relevant for this object. Taken together, these data suggest an important role of flexibility and local deformations in DNA origami objects, which has to be considered when designing complex origami structures.

In summary, we have demonstrated the ability for SAXS to sensitively monitor conformational changes of self-assembled DNA origami objects in solution. SAXS provides a number of advantages: First, being a solution-based technique, SAXS is free of potential 
biases and perturbations from the proximity of a surface. Second, SAXS is a label free method, without the need to chemically modify the structure of interest. Third, SAXS reads out the global conformation of molecules or molecular assemblies in solution, as defined by their electron density, thus avoiding concerns whether e.g. variations in fluorescence might stem from local conformational changes or photophysical effects upon changes in solution condition. Taken together, these advantages render SAXS a very promising novel approach for detecting conformational states of dynamic DNA origami objects and we anticipate that many of the techniques' capabilities that were previously demonstrated in other contexts can be extended towards monitoring conformational changes in DNA nanostructures, including temperature controlled ${ }^{74}$ and/or time-resolved $\mathrm{SAXS}^{31,32,75}$ measurements and the detection and characterization of structural intermediates and molecular ensembles. ${ }^{36,39,76}$

Quantitative comparison of the experimental SAXS data to theoretical profiles derived from 3D models of the DNA objects reveal considerable flexibility and deformations away from the idealized "design" structure. Such deformations will have to be taken into account for high-resolution designs in the future. In addition, this work highlights the ability of SAXS to critically test structural models against solution-based data, even for very large DNA objects, which constitutes a promising approach that is complementary to the more routinely used methods.

\section{ASSOCIATED CONTENT}

Supplementary Methods, Table S1, and Supplementary Figures S1-S13. This material is available free of charge via the Internet at http://pubs.acs.org.

\section{AUTHOR INFORMATION}

\section{Corresponding Author}

Jan Lipfert; Email: Jan.Lipfert@1mu.de; Phone: +49-80-2180-2005 


\section{Author Contributions}

L.K.B., T.G., H.D., and J.L. designed the study; T.G. assembled and purified samples; L.K.B., S.M.S., and P.W. performed SAXS measurements; W.Z. performed structure refinement. All authors analyzed data, contributed to writing the paper, and have given approval to the final version of the manuscript.

\section{Notes}

The authors declare no competing financial interest.

\section{FUNDING}

This work was supported by the Deutsche Forschungsgemeinschaft through grants provided within Gottfried-Wilhelm Leibniz Program, the Excellence Clusters CIPSM (Center for Integrated Protein Science Munich), NIM (Nanosystems Initiative Munich), and the Sonderforschungsbereich SFB863.

\section{ACKNOWLEDGEMENTS}

We thank Dr. Keyao Pan and Dr. Marc Delarue for helpful discussions and support on atomistic modeling and normal mode analysis and Dr. Adam Round for assistance at beamline BM29 at the ESRF (Grenoble, France). Moreover, we acknowledge Dr. Alessandro Spilotros and Nelly Hajizadeh for help at beamline P12 at DESY (Hamburg,Germany).

\footnotetext{
ABBREVIATIONS

SAXS, small-angle X-ray scattering; FRET, (Förster) fluorescence resonance energy transfer; AFM, atomic force microscopy; Switch C, closed switch; switch O, open switch; switch D, dynamic switch.
} 


\section{TABLES}

Table 1. Comparison of the radius of gyration $\left(R_{g}\right)$ and the cross-sectional $R_{g}\left(R_{c}\right)$ for the static and dynamic versions of the switch object derived from experimental and theoretical scattering profiles. Experimental data correspond to averaged results from concentration scaled scattering profiles for sample concentrations of 25, 50 and $100 \mathrm{nM}$. "Values were determined from Guinier fits of the predicted scattering profiles in the fitting range $q_{\max } \cdot R_{g}<1.3$.

\begin{tabular}{lcc}
\hline \multicolumn{1}{c}{ Sample } & $\mathbf{R}_{\mathrm{g}}(\mathbf{n m})$ & $\mathbf{R}_{\mathbf{c}}(\mathbf{n m})$ \\
\hline Switch C & $27.9( \pm 0.1)$ & $6.7( \pm 0.1)$ \\
Switch O & $29( \pm 0.2)$ & $4.8( \pm 0.0)$ \\
Switch D30 & $28.1( \pm 0.1)$ & $6.0( \pm 0.1)$ \\
Switch D05 & $27.5( \pm 0.2)$ & $4.8( \pm 0.0)$ \\
CRYSOL (closed) & $28.2^{*}$ & 6.4 \\
CRYSOL (open) & $29.5^{*}$ & 4.4 \\
\hline \hline
\end{tabular}




\section{FIGURES}

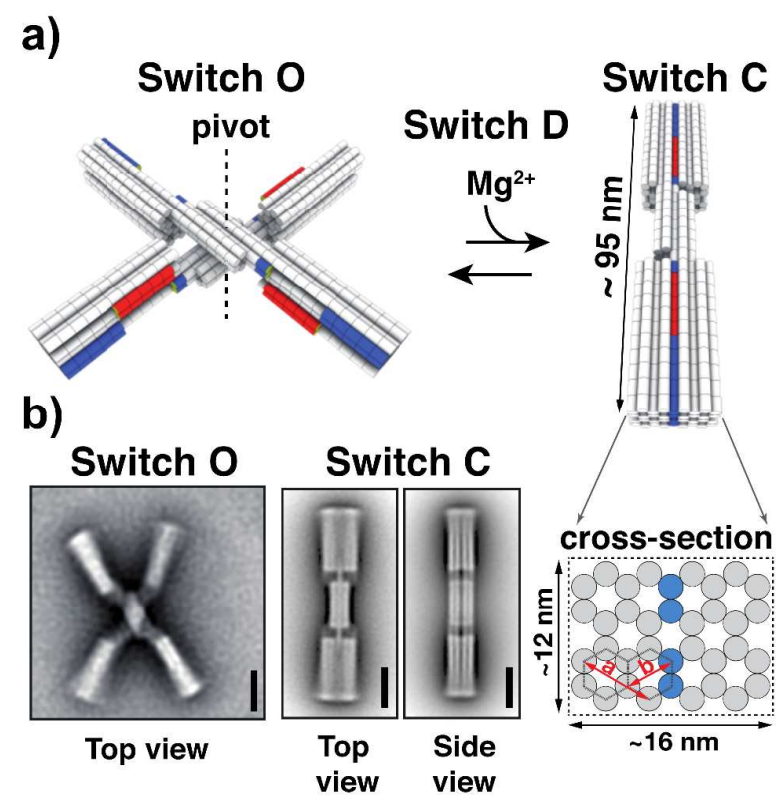

Figure 1. Illustrations of static and dynamic switch devices.(a) Schematics of the switch devices used in this study. The dynamic switch object (switch D) changes from an X-shaped open to a rectangular-shaped closed conformation upon addition of magnesium ions. Shapecomplementary protrusions and recessions are indicated by the red and blue DNA double helical domains, respectively. Static switch variants are locked in the open (switch O, left) and closed (switch $\mathrm{C}$, right) state. The schematic of the cross-sectional area of switch $\mathrm{C}$ indicates the horizontal and vertical dimensions including inter-helical distances of $a=6 \mathrm{~nm}$ and $b=4 \mathrm{~nm}$, which give rise to a peak in the scattering profiles of switch $\mathrm{C}$ and switch $\mathrm{O}$. (b) Corresponding average negative-stain TEM micrographs of switch $\mathrm{O}$ in the presence of $5 \mathrm{mM}$ $\mathrm{MgCl}_{2}$ and of switch $\mathrm{C}$ at a $\mathrm{MgCl}_{2}$ concentration of $25 \mathrm{mM}$. Scale bars, $20 \mathrm{~nm}$. 


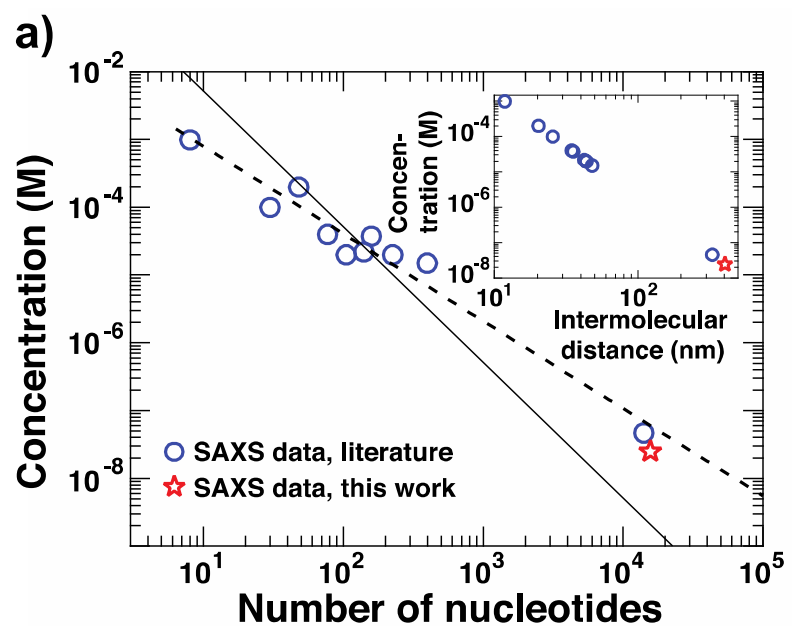

b)

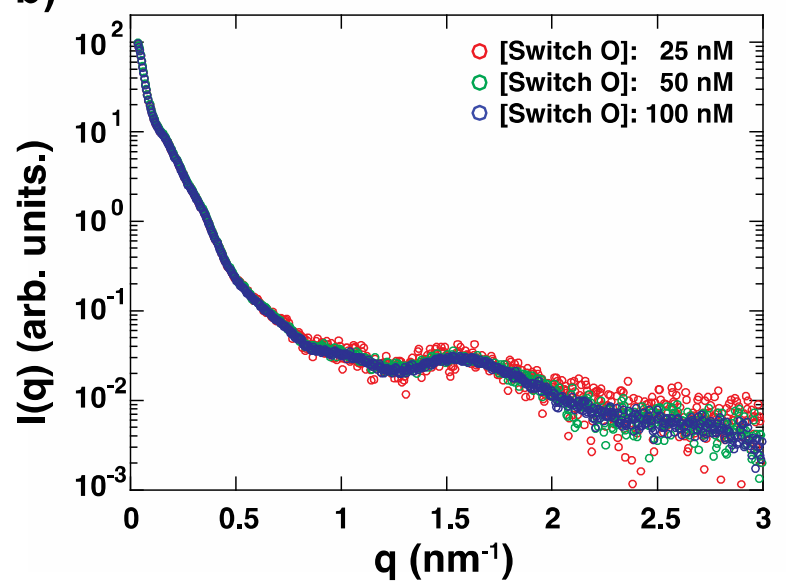

Figure 2. Concentration requirements and SAXS signals for large DNA origami structures. (a) Concentrations required to obtain a suitable SAXS signal, as a function of molecule size (in number of nucleotides), for a range of nucleic acid samples investigated previously (blue circles). The solid line is a fit of the relationship $a / n t^{2}$, where $n t$ is the number of nucleotides and $a$ a fitting constant. The dashed line is a fit of the relationship $b / n t^{v}$ where $b$ and $v$ are fitting constants. From the best fit we find $v \sim 1.30$. The red star corresponds to measurements of the DNA origami switch samples in this study that were guided by the scaling behavior. Inset: Intermolecular distances calculated for the required SAXS concentrations of the different nucleic acid samples. (b) Averaged scattering profiles of the switch $\mathrm{O}$ measured at three different concentrations: $25 \mathrm{nM}$ (red circles), $50 \mathrm{nM}$ (green circles), $100 \mathrm{nM}$ (blue circles). Data are scaled by concentration. 

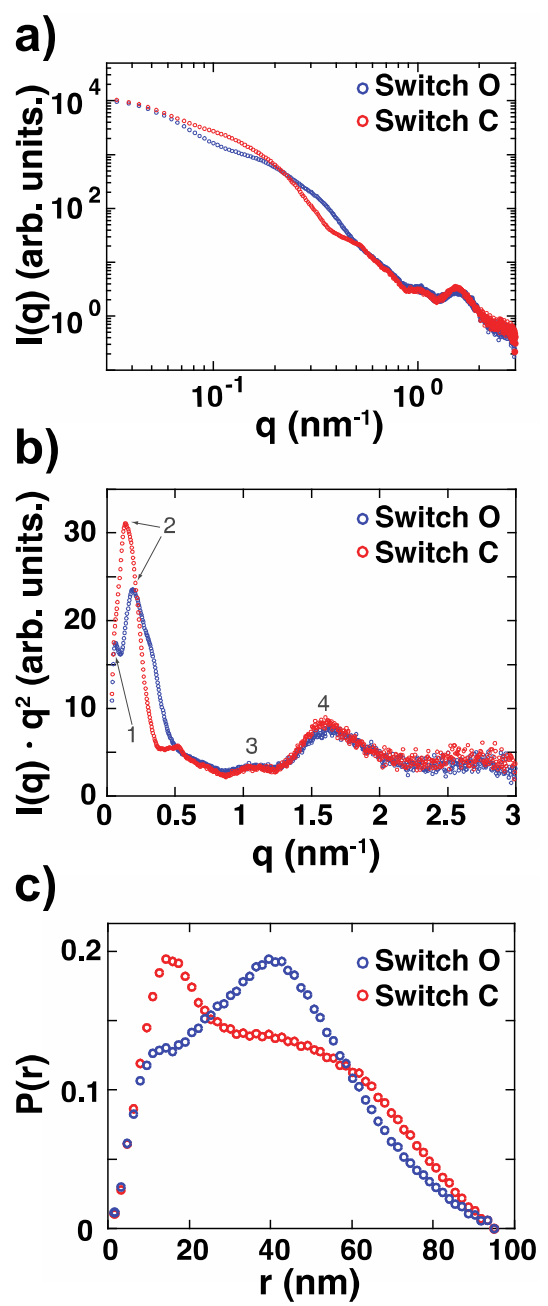

Figure 3. SAXS data reveal conformational features for open and closed switch objects. (a) Double-logarithmic representation of scattering intensity profiles obtained from the switch $\mathrm{O}$ and switch C sample. (b) Kratky representation of the data from (a) scaled by a constant factor. Numbers indicate peaks, which are described in the main text. (c) Pair distance distribution function $P(r)$ calculated from data shown in (a) assuming a maximum particle dimension $D_{\max }$ of $95 \mathrm{~nm} . P(r)$ functions are normalized to equal areas. 
a)

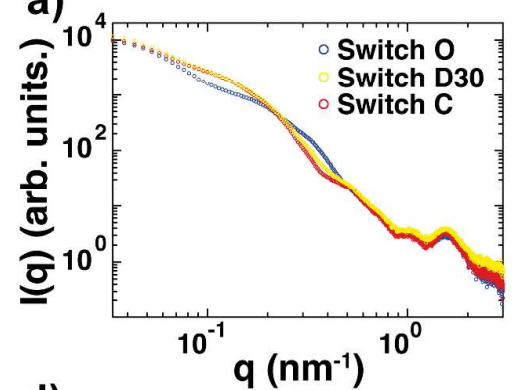

d)

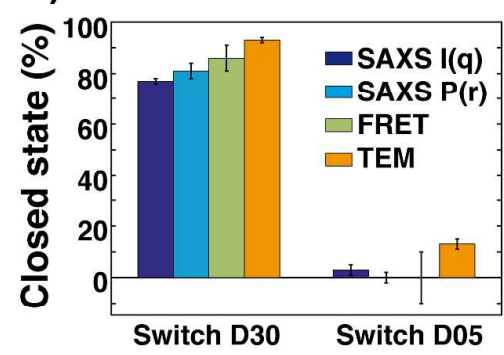

b)

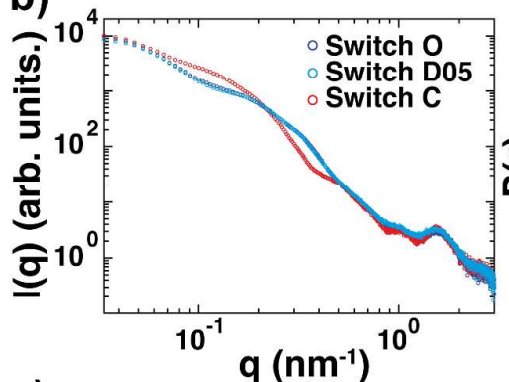

e)

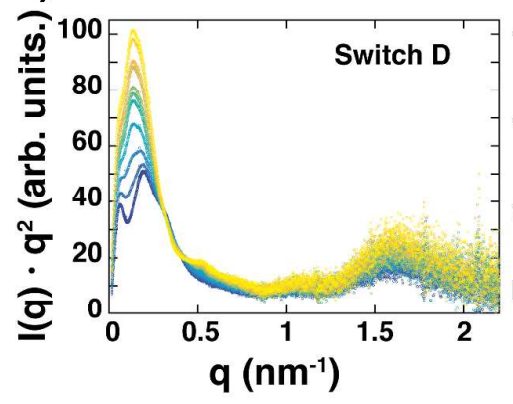

c)

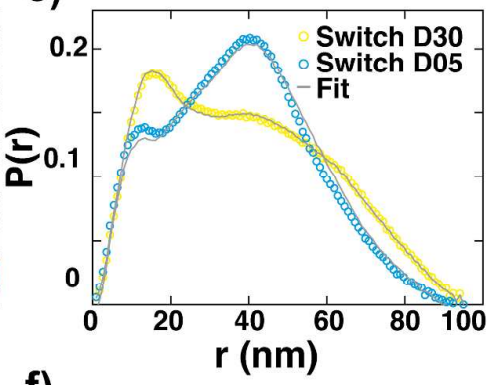

f)

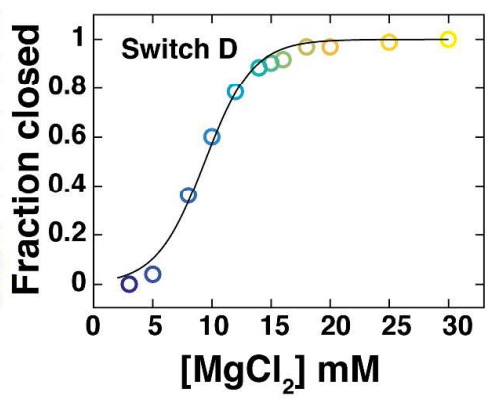

Figure 4. Characterization of conformational states of dynamic switch structures. (a) Comparison of the scattering profile from the switch D30 (yellow) to the scattering profiles of the static switch samples switch $\mathrm{O}$ (blue) and switch $\mathrm{C}$ (red). (b) Scattering profile from the switch D05 sample (cyan) in comparison to scattering curves from the static structures (same color code as in (a)). (c) $P(r)$ functions of the dynamic switch variants (cyan, yellow circles) and the resulting two-state model fits (grey lines). (d) Comparison of the relative fractions of the closed states determined from the scattering profiles (blue bars), the $P(r)$ functions (cyan bars), ensemble FRET (green bars), and TEM imaging (orange bars) for the switch D30 and switch D05 samples, corresponding to $\mathrm{MgCl}_{2}$ concentrations of $30 \mathrm{mM}$ and $5 \mathrm{mM}$, respectively. For TEM imaging the highest $\mathrm{MgCl}_{2}$ concentration was $25 \mathrm{mM}$. (e) Kratky representation of the scattering profiles of switch $\mathrm{D}$ samples for varying $\mathrm{MgCl}_{2}$ concentrations: $3 \mathrm{mM}$ (dark blue,bottom), $5 \mathrm{mM}, 8 \mathrm{mM}, 10 \mathrm{mM}, 12 \mathrm{mM}, 14 \mathrm{mM}, 15 \mathrm{mM}$, $16 \mathrm{mM}, 18 \mathrm{mM}, 20 \mathrm{mM}, 25 \mathrm{mM}$ and $30 \mathrm{mM}$ (light yellow, top). Data are normalized to the intensity at zero scattering angle and scaled by a constant factor. (f) Fraction of closed switch particles for $\mathrm{MgCl}_{2}$ titration experiments shown in (e), determined from a two-state model. Solid lines represent a two-state model with a free energy term that depends linearly on the $\mathrm{MgCl}_{2}$ concentration. 
a) b)

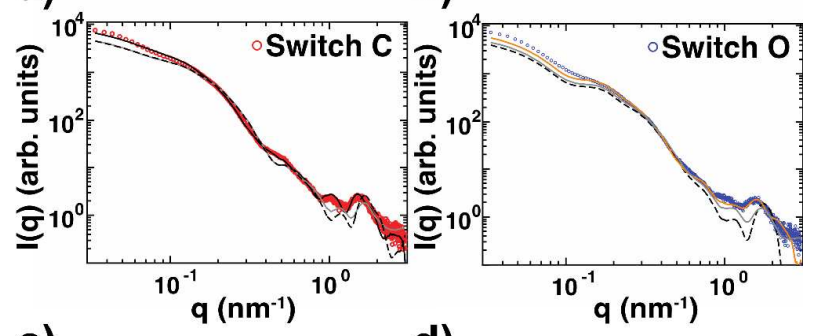

c)

d)

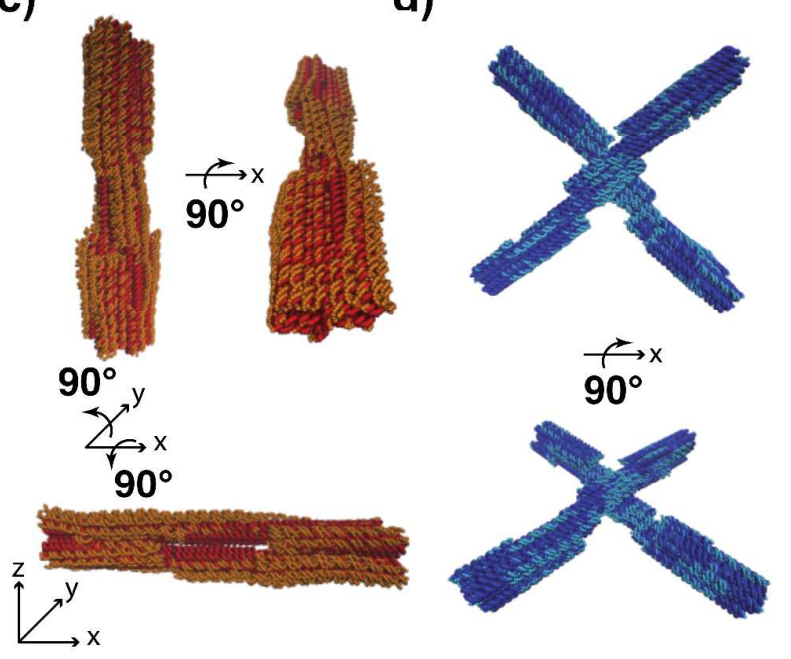

Figure 5. Normal mode based refinement of DNA origami structures against SAXS data. (a) shows data for the switch $\mathrm{C}$ construct and (b) the corresponding results for the switch $\mathrm{O}$ sample. Experimental scattering profiles are shown as red or blue circles. Scattering profiles predicted from the initial, CanDo derived models using all atoms and the software CRYSOL are shown as grey lines and using a one-bead-per-base representation as dashed black lines. Scattering profiles for the final models (computed using the one-bead-per-base representation) after normal mode based refinement are shown as black (for switch $\mathrm{C}$ ) and orange (for switch O) lines. (c) and (d) show the initial models for the switch $\mathrm{C}$ and switch $\mathrm{O}$ objects as red and blue tubes and the final models after normal mode refinement as orange and cyan spheres, respectively. 


\section{REFERENCES}

(1) Seeman, N. C. Nature 2003, 421 (6921), 427-431.

(2) Rothemund, P. W. K. Nature 2006, 440 (7082), 297-302.

(3) Winfree, E.; Liu, F.; Wenzler, L. A.; Seeman, N. C. Nature 1998, 394 (6693), 539544.

(4) Castro, C. E.; Kilchherr, F.; Kim, D.-N.; Shiao, E. L.; Wauer, T.; Wortmann, P.; Bathe, M.; Dietz, H. Nature Methods 2011, 8 (3), 221-229.

(5) Douglas, S. M.; Dietz, H.; Liedl, T.; Högberg, B.; Graf, F.; Shih, W. M. Nature 2009, 459 (7245), 414-418.

(6) Dietz, H.; Douglas, S. M.; Shih, W. M. Science 2009, 325 (5941), 725-730.

(7) Benson, E.; Mohammed, A.; Gardell, J.; Masich, S.; Czeizler, E.; Orponen, P.; Högberg, B. Nature 2015, 523 (7561), 441-U139.

(8) Ke, Y.; Ong, L. L.; Shih, W. M.; Yin, P. Science 2012, 338 (6111), 1177-1183.

(9) Andersen, E. S.; Dong, M.; Nielsen, M. M.; Jahn, K.; Subramani, R.; Mamdouh, W.; Golas, M. M.; Sander, B.; Stark, H.; Cristiano L. P. Oliveira; Pedersen, J. S.; Birkedal, V.; Besenbacher, F.; Gothelf, K. V.; Kjems, J. Nature 2009, 459 (7243), 73-76.

(10) Douglas, S. M.; Bachelet, I.; Church, G. M. Science 2012, 335 (6070), 831-834.

(11) Kuzyk, A.; Schreiber, R.; Zhang, H.; Govorov, A. O.; Liedl, T.; Liu, N. Nature Materials 2014, 13 (9), 862-866.

(12) Liu, M.; Fu, J.; Hejesen, C.; Yang, Y.; Woodbury, N. W.; Gothelf, K.; Liu, Y.; Yan, H. Nature Communications 2013, 4.

(13) Zhang, D. Y.; Seelig, G. Nat Chem 2011, 3 (2), 103-113.

(14) Marras, A. E.; Zhou, L.; Su, H.-J.; Castro, C. E. PNAS 2015, 112 (3), 713-718.

(15) Kuzuya, A.; Ohya, Y. Acc. Chem. Res. 2014, 47 (6), 1742-1749.

(16) Zhao, Y.-X.; Shaw, A.; Zeng, X.; Benson, E.; Nyström, A. M.; Högberg, B. ACS Nano 2012, 6 (10), 8684-8691.

(17) Zhang, F.; Jiang, S.; Wu, S.; Li, Y.; Mao, C.; Liu, Y.; Yan, H. Nature Nanotechnology 2015, 10 (9), 779-784.

(18) Aghebat Rafat, A.; Pirzer, T.; Scheible, M. B.; Kostina, A.; Simmel, F. C. Angew. Chem. Int. Ed. Engl. 2014, 53 (29), 7665-7668.

(19) Langecker, M.; Arnaut, V.; Martin, T. G.; List, J.; Renner, S.; Mayer, M.; Dietz, H.; Simmel, F. C. Science 2012, 338 (6109), 932-936.

(20) Han, D.; Pal, S.; Nangreave, J.; Deng, Z.; Liu, Y.; Yan, H. Science 2011, 332 (6027), 342-346.

(21) Schreiber, R.; Luong, N.; Fan, Z.; Kuzyk, A.; Nickels, P. C.; Zhang, T.; Smith, D. M.; Yurke, B.; Kuang, W.; Govorov, A. O.; Liedl, T. Nature Communications 2013, 4, 2948.

(22) Bai, X.-C.; Martin, T. G.; Scheres, S. H. W.; Dietz, H. PNAS 2012, 109 (49), 2001220017.

(23) Koch, M. H. J.; Vachette, P.; Svergun, D. I. Quarterly Reviews of Biophysics 2003, $36(02), 147-227$.

(24) Lipfert, J.; Doniach, S. Annu Rev Biophys Biomol Struct 2007, 36 (1), 307-327.

(25) Rambo, R. P.; Tainer, J. A. Annu Rev Biophys 2013, 42 (1), 415-441.

(26) Putnam, C. D.; Hammel, M.; Hura, G. L.; Tainer, J. A. Quarterly Reviews of Biophysics 2007, 40 (3), 191-285.

(27) Eliezer, D.; Jennings, P. A.; Wright, P. E.; Doniach, S.; Hodgson, K. O.; Tsuruta, H. Science 1995, 270 (5235), 487-488.

(28) Akiyama, S.; Takahashi, S.; Kimura, T.; Ishimori, K.; Morishima, I.; Nishikawa, Y.; Fujisawa, T. PNAS 2002, 99 (3), 1329-1334. 
(29) Doniach, S. Chem. Rev. 2001, 101 (6), 1763-1778.

(30) Fang, X.; Littrell, K.; Yang, X.-J.; Henderson, S. J.; Siefert, S.; Thiyagarajan, P.; Pan, T.; Sosnick, T. R. Biochemistry 2000, 39 (36), 11107-11113.

(31) Russell, R.; Millett, I. S.; Tate, M. W.; Kwok, L. W.; Nakatani, B.; Gruner, S. M.; Mochrie, S. G. J.; Pande, V.; Doniach, S.; Herschlag, D.; Pollack, L. Proc. Natl. Acad. Sci. U.S.A. 2002, 99 (7), 4266-4271.

(32) Herschlag, D.; Russell, R.; Millett, I. S.; Doniach, S. Nat. Struct Biol. 2000, 7 (5), 367-370.

(33) Tuukkanen, A. T.; Svergun, D. I. FEBS J. 2014, 281 (8), 1974-1987.

(34) Bessman, N. J.; Bagchi, A.; Ferguson, K. M.; Lemmon, M. A. Cell Rep 2014, 9 (4), 1306-1317.

(35) Rambo, R. P.; Tainer, J. A. RNA 2010, 16 (3), 638-646.

(36) Lipfert, J.; Das, R.; Chu, V. B.; Kudaravalli, M.; Boyd, N.; Herschlag, D.; Doniach, S. J. Mol. Biol. 2007, 365 (5), 1393-1406.

(37) Gerling, T.; Wagenbauer, K. F.; Neuner, A. M.; Dietz, H. Science 2015, 347 (6229), 1446-1452.

(38) Sim, A. Y. L.; Lipfert, J.; Herschlag, D.; Doniach, S. Phys. Rev. E 2012, 86 (2), 021901.

(39) Ali, M.; Lipfert, J.; Seifert, S.; Herschlag, D.; Doniach, S. J. Mol. Biol. 2010, 396 (1), $153-165$.

(40) Lipfert, J.; Ouellet, J.; Norman, D. G.; Doniach, S.; Lilley, D. M. J. Structure 2008, $16(9), 1357-1367$.

(41) Bai, Y.; Das, R.; Millett, I. S.; Herschlag, D.; Doniach, S. Proc. Natl. Acad. Sci. U.S.A. 2005, 102 (4), 1035-1040.

(42) Meisburger, S. P.; Sutton, J. L.; Chen, H.; Pabit, S. A.; Kirmizialtin, S.; Elber, R.; Pollack, L. Biopolymers 2013, 99 (12), 1032-1045.

(43) Kratky, O.; Glatter, O. Academic Press, London 1982, 1-267.

(44) Zuo, X.; Cui, G.; Merz, K. M.; Zhang, L.; Lewis, F. D.; Tiede, D. M. Proc. Natl. Acad. Sci. U.S.A. 2006, 103 (10), 3534-3539.

(45) Chiu, T. K.; Dickerson, R. E. J. Mol. Biol. 2000, 301 (4), 915-945.

(46) Martin, T. G.; Dietz, H. Nature Communications 2012, 3, 1103.

(47) Frank, J. Annu Rev Biophys Biomol Struct 2002, 31 (1), 303-319.

(48) Hura, G. L.; Menon, A. L.; Hammel, M.; Rambo, R. P.; Poole, F. L., II; Tsutakawa, S. E.; Jenney, F. E., Jr; Classen, S.; Frankel, K. A.; Hopkins, R. C.; Yang, S.-J.; Scott, J. W.; Dillard, B. D.; Adams, M. W. W.; Tainer, J. A. Nature Methods 2009, 6 (8), 606-612.

(49) Svergun, D.; Barberato, C.; Koch, M. J Appl Crystallogr 1995, 28 (6), 768-773.

(50) Schneidman-Duhovny, D.; Hammel, M.; Sali, A. Nucl. Acids Res. 2010, 38 (Web Server issue), W540-W544.

(51) Poitevin, F.; Orland, H.; Doniach, S.; Koehl, P.; Delarue, M. Nucl. Acids Res. 2011, 39 (Web Server issue), W184-W189.

(52) Ravikumar, K. M.; Huang, W.; Yang, S. The Journal of Chemical Physics 2013, 138 (2), 024112-024118.

(53) Pan, K.; Kim, D.-N.; Zhang, F.; Adendorff, M. R.; Yan, H.; Bathe, M. Nature Communications 2014, 5, 5578-7.

(54) Svergun, D. I.; Richard, S.; Koch, M. H.; Sayers, Z.; Kuprin, S.; Zaccai, G. Proc. Natl. Acad. Sci. U.S.A. 1998, 95 (5), 2267-2272.

(55) Pollack, L. Annu Rev Biophys 2011, 40 (1), 225-242.

(56) Lipfert, J.; Doniach, S.; Das, R.; Herschlag, D. Annu. Rev. Biochem. 2014, 83 (1), 813-841.

(57) Meisburger, S. P.; Pabit, S. A.; Pollack, L. Biophysj 2015, 108 (12), 2886-2895. 
(58) Gavryushov, S.; Zielenkiewicz, P. Biophysj 1998, 75 (6), 2732-2742.

(59) Yoo, J.; Aksimentiev, A. Biophysj 2014, 106 (2), 695a.

(60) Kim, D. N.; Kilchherr, F.; Dietz, H.; Bathe, M. Nucl. Acids Res. 2012, 40 (7), 28622868.

(61) Yoo, J.; Aksimentiev, A. PNAS 2013, 110 (50), 20099-20104.

(62) Sedeh, R. S.; Pan, K.; Adendorff, M. R.; Hallatschek, O.; Bathe, K.-J.; Bathe, M. J Chem Theory Comput 2016, 12 (1), 261-273.

(63) Maffeo, C.; Yoo, J.; Aksimentiev, A. Nucl. Acids Res. 2016, gkw155.

(64) Miyashita, O.; Gorba, C.; Tama, F. Journal of Structural Biology 2011, 173 (3), 451460.

(65) López-Blanco, J. R.; Miyashita, O.; Tama, F.; Chacón, P. Normal Mode Analysis Techniques in Structural Biology; John Wiley \& Sons, Ltd: Chichester, UK, 2001; pp $1-12$.

(66) Tama, F.; Sanejouand, Y. H. Protein Eng. 2001, 14 (1), 1-6.

(67) Delarue, M. Acta Crystallogr Sect D Biol Crystallogr 2008, 64 (Pt 1), 40-48.

(68) Hinsen, K. Proteins 1998, 33 (3), 417-429.

(69) Tama, F.; Valle, M.; Frank, J.; Brooks, C. L. Proc. Natl. Acad. Sci. U.S.A. 2003, 100 (16), 9319-9323.

(70) Schröder, G. F.; Levitt, M.; Brunger, A. T. Nature 2010, 464 (7292), 1218-1222.

(71) Gorba, C.; Tama, F. Bioinform Biol Insights 2010, 4, 43-54.

(72) Zheng, W.; Tekpinar, M. Biophys. J. 2011, 101 (12), 2981-2991.

(73) Panjkovich, A.; Svergun, D. I. Physical Chemistry Chemical Physics 2016, 18 (8), 5707-5719.

(74) Pérez, J.; Vachette, P.; Russo, D.; Desmadril, M.; Durand, D. J. Mol. Biol. 2001, 308 (4), 721-743.

(75) Pollack, L. Biopolymers 2011, 95 (8), 543-549.

(76) Baird, N. J.; Westhof, E.; Qin, H.; Pan, T.; Sosnick, T. R. J. Mol. Biol. 2005, 352 (3), 712-722. 


\section{FOR TOC ONLY}

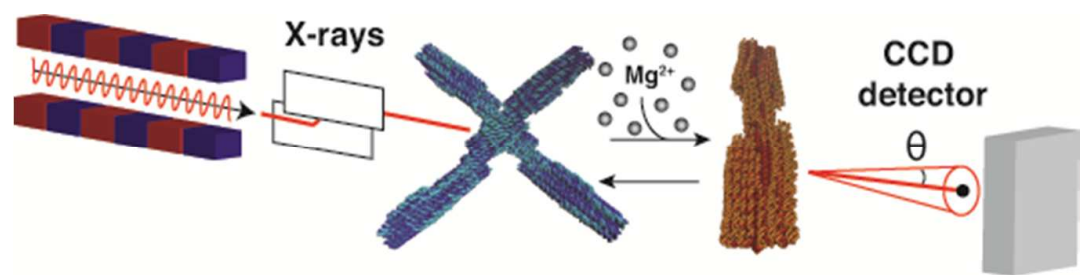

